\title{
Improvement of the Rett Syndrome Phenotype in a Mecp2 Mouse Model Upon Treatment with Levodopa and a Dopa-Decarboxylase Inhibitor
}

\author{
Karolina Szczesna', Olga de la Caridad', Paolo Petazzi', Marta Soler', Laura Roa', Mauricio A Saez', \\ Stéphane Fourcade ${ }^{2,3,4}$, Aurora Pujol ${ }^{2,3,4,5}$, Rafael Artuch-Iriberri ${ }^{4,6}$, Marta Molero-Luis ${ }^{4,6}$, August Vidal ${ }^{7}$, \\ Dori Huertas*,1 and Manel Esteller*,1,5,8
}

'Cancer Epigenetics and Biology Program (PEBC), Bellvitge Biomedical Research Institute (IDIBELL), Barcelona, Spain; ${ }^{2}$ Neurometabolic Diseases Laboratory, Bellvitge Biomedical Research Institute (IDIBELL), Barcelona, Spain; ${ }^{3}$ nnstitute of Neuropathology, University of Barcelona, Barcelona, Spain; ${ }^{4}$ Center for Biomedical Research on Rare Diseases (CIBERER), ISCIII, Madrid, Spain; Institucio Catalana de Recerca i Estudis Avançats (ICREA), Barcelona, Spain; ${ }^{6}$ Neurometabolic Unit, Hospital Sant Joan de Déu, Barcelona, Spain; ${ }^{7}$ Department of Pathology, Bellvitge University Hospital, Bellvitge Biomedical Research Institute (IDIBELL), Barcelona, Spain; ${ }^{8}$ Department of Physiological Sciences II, School of Medicine, University of Barcelona, Barcelona, Spain

\begin{abstract}
Rett Syndrome is a neurodevelopmental autism spectrum disorder caused by mutations in the gene coding for methyl CpG-binding protein (MeCP2). The disease is characterized by abnormal motor, respiratory, cognitive impairment, and autistic-like behaviors. No effective treatment of the disorder is available. Mecp2 knockout mice have a range of physiological and neurological abnormalities that resemble the human syndrome and can be used as a model to interrogate new therapies. Herein, we show that the combined administration of Levodopa and a Dopa-decarboxylase inhibitor in RTT mouse models is well tolerated, diminishes RTT-associated symptoms, and increases life span. The amelioration of RTT symptomatology is particularly significant in those features controlled by the dopaminergic pathway in the nigrostratium, such as mobility, tremor, and breathing. Most important, the improvement of the RTT phenotype upon use of the combined treatment is reflected at the cellular level by the development of neuronal dendritic growth. However, much work is required to extend the duration of the benefit of the described preclinical treatment.

Neuropsychopharmacology (2014) 39, 2846-2856; doi:I0.1038/npp.20I4.136; published online 9 July 2014
\end{abstract}

\section{INTRODUCTION}

Mutations in the $\mathrm{X}$-linked $M E C P 2$ gene are the primary cause of Rett syndrome (RTT; OMIM no. 312750), a neurodevelopmental autism spectrum disorder with delayed onset that affects 1 in 10000 girls (Amir et al, 1999). Mutations most frequently occur sporadically, and familiar cases are rare. Patients with RTT appear to develop normally up to 6-18 months of age. The clinical features of RTT include small hands and feet, and a decelerated rate of head growth (Neul and Zoghbi, 2004). RTT patients show abnormal neuronal morphology but not neuronal death (Armstrong, 1995), which implies that RTT is a neurodevelopmental, rather than a neurodegenerative disorder. MeCP2 is widely expressed, but it is most abundant in

*Correspondence: Dr D Huertas or Dr M Esteller, Cancer Epigenetics and Biology Program (PEBC), Bellvitge Biomedical Research Institute (IDIBELL), 3rd Floor, Hospital Duran i Reynals, Avenue Gran Via 199-203, L'Hospitalet, Barcelona 08908, Catalonia, Spain, Tel: +34 932607253, Fax:+34932607|40, E-mail: dhuertas@idibell.cat or mesteller@idibell.cat Received 3 February 2014; revised 28 May 2014; accepted 2 June 2014; accepted article preview online II June 2014 neurons of the mature nervous system (Kishi and Macklis, 2004).

The brain and cerebrospinal fluid analyses performed in RTT patients showed reduced levels of dopamine (Riderer et al, 1986; Lekman et al, 1989; Wenk et al, 1991). A low frequency of dopamine 2 receptors (D2R) in the brain of an RTT girl have been described (Chiron et al, 1993), and subsequently concluded as being a possible cause for low numbers of spines (Fasano et al, 2013). Neuropathology also revealed a reduction in the number of neurons in the substantia nigra (Kitt and Wilcox, 1995), and a reduction in tyrosine hydroxylase (Th) immunoreactivity (Jellinger et al, 1988). An abnormal thinning of dendrites within the substantia nigra in RTT girls was also described (Jellinger, 2003). An alteration of dopaminergic metabolism may also be involved in the abnormal motor movements and late motor deterioration that are observed in the classic form of RTT (FitzGerald et al, 1990).

The symptoms of RTT are caused by a neuronal MeCP2 deficiency, and expression of $\mathrm{MeCP} 2$ in postmitotic neurons rescues RTT in mice (Luikenhuis et al, 2004). The mouse model we studied harbors a deletion of Mecp2 exons 3 and 4 , which encode the methyl-binding domain and the 
transcription repression domain, respectively (Guy et al, 2001). As previously described not only in RTT patients but also in RTT model mice, the levels of norepinephrine and dopamine are reduced (Ide et al, 2005) and Th-expressing neurons are deficient (Viemari et al, 2005). A deficiency in the midbrain (MB) of catecholaminergic metabolism in Mecp2 knockout (KO) mice has also been described (Samaco et al, 2009). The MB dopaminergic (mDA) area substantia nigra pars compacta $(\mathrm{SNpc})$ regulates the production of motor strategies (Blandini et al, 2000), and dopaminergic deficit is also the cause of motor deficits in Parkinson disease (Jenner, 2008). In this regard, the mature dendritic arbors of pyramidal neurons are severely retracted, and dendritic spine density is markedly reduced in Mecp2 KO mice (Nguyen et al, 2012). Most importantly, it has already been demonstrated that a conditional loss of Mecp2 in brain areas induces motor impairments and dopaminergic deficits in the SNpc of Mecp2 KO mice. Most strikingly, when L-Dopa treatment is implemented, the behavioral effects of Mecp2 KO are improved (Panayotis et al, 2011).

Following the dopaminergic deficiency in RTT described above, and the usual combination of L-Dopa with a dopadecarboxylase inhibitor (Ddci) to prevent the conversion of levodopa into dopamine in the peripheral circulation (Olanow et al, 2001) and to provide an efficient dopaminergic stimulation therapy in Parkinsonism and related disorders (Mena et al, 2009), we wondered whether the same type of dual approach could help ameliorate the clinicopathological features of the Mecp2 null mouse. We observed that the combined administration of L-Dopa and the Ddci benserazide was able to improve the mobility, tremor, and breathing phenotype of the Mecp2 KO mice in association with an increase in dopaminergic activity and dendritic spine density.

\section{MATERIALS AND METHODS}

\section{Animals}

Experiments were performed on the B6.129P2(c)Mecp2tm1 + 1Bird mouse model for RTT (Guy et al, 2001). The exact strain used was C57BL/6J and it was maintained by backcrossing with C57BL/6J purchased from The Jackson Laboratory. Both pre-symptomatic and symptomatic mice were analyzed at different developmental stages. A total of 157 littermate male mice were used in the study divided in five groups: Mecp2 wild-type (WT; $n=30$ ), Mecp2-/y vehicle-treated $(n=39)$, Mecp2-/y Ddci-treated $(n=15)$, Mecp2-/y L-Dopa-treated $(n=30)$, and Mecp2-/y L-Dopa + Ddci-treated $(n=43)$. Animals used for the experiments were all males and ranged in age from 4 to 10 weeks. All mice procedures and experiments were approved by the Ethics Committee for Animal Experiments of the IDIBELL Centre, under the guidelines set out under Spanish animal welfare laws. For the pharmacological treatments, treated and untreated (vehicle) mice were studied. Mecp2-deficient treated and untreated mice were compared with their respective WT littermates. The Mecp 2 - /y (null male) and WT (male) mice were subjected to immunohistochemistry, neurochemical analysis, and western blot studies at 24,55 , and 70 postnatal days. For the pharmacological treatments, Mecp2 KO mice received for 6 weeks a once-a-day dose of intraperitoneal Ddci (12 mg/kg/day, Benserazide, B7283; Sigma-Aldrich) in saline vehicle, L-Dopa (30 mg/kg/day, Sigma-Aldrich; D1507) or L-Dopa + Ddci. In the L-Dopa + Ddci group, the Ddci drug was injected 15 min before L-Dopa administration.

\section{Score Test}

The score was based on that previously adopted in the study of the Mecp2 null mouse model (Guy et al, 2001; Guy et al, 2007). The treatment started when animals were 4 weeks old. At that time, it was still not possible to observe characteristic symptoms of this model. Every day, mice were weighed in order to apply the appropriate dose of the injection. Every 2 days (the starting point being the first day of treatment), neurological defects in an MeCP2 mouse model were scored (blind to genotype and treatment; twice a week, at the same time of day), focusing on mobility, gait, hindlimb clasping, tremor, breathing, and general condition. Each of the six symptoms was scored from 0 to $2 ; 0$ corresponds to the symptom being absent or the same as in the WT animal, 1 where the symptom was present, and 2 when the symptom was severe. In detail:

(A) Mobility: the mouse is observed when placed on bench, then when handled gently. $0=$ As WT. $1=$ Reduced movement when compared with WT: extended freezing period when first placed on bench and longer periods spent immobile. $2=$ No spontaneous movement when placed on the bench; mouse can move in response to a gentle prod or a food pellet placed nearby.

(B) Gait: $0=$ As WT. $1=$ Hind legs are spread wider than WT when walking or running with reduced pelvic elevation, resulting in a 'waddling' gait. $2=$ More severe abnormalities: tremor when feet are lifted, walks backward or 'bunny hops' by lifting both rear feet at once.

(C) Hindlimb clasping: mouse observed when suspended by holding base of the tail. $0=$ Legs splayed outward. $1=$ Hindlimbs are drawn toward each other (without touching) or one leg is drawn into the body. $2=$ Both legs are pulled in tightly, either touching each other or touching the body.

(D) Tremor: mouse observed while standing on the flat palm of the hand. $0=$ No tremor. $1=$ Intermittent mild tremor. $2=$ Continuous tremor or intermittent violent tremor.

(E) Breathing: movement of flanks observed while animal is standing still. $0=$ Normal breathing. $1=$ Periods of regular breathing interspersed with short periods of more rapid breathing or with pauses in breathing. $2=$ Very irregular breathing-gasping or panting.

(F) General condition: mouse observed for indicators of general well-being such as coat condition, eyes, and body stance. $0=$ Clean shiny coat, clear eyes, and normal stance. $1=$ Eyes dull, coat dull/ungroomed, and somewhat hunched stance. 2 = Eyes crusted or narrowed, piloerection, and hunched posture.

If at any point an animal scored 2 out of 2 for the last three criteria, or if the animal lost $20 \%$ of its body weight during the experiment, it was killed. Inter-rater reliability for each qualitative parameter was assessed using a Cohen's Kappa statistic (Kappa) that controls for chance agreement between two raters (study rater and an experienced rater), with a value of 1 indicating perfect agreement, and 
performed for all the individuals. All the parameters show Kappa $>0.7$ and associated $p$-value $<0.05$. For the measure of Cohen's Kappa statistics was used using irr package (Gamer et al, 2012) under R statistical software (R Core Team, 2013). The two observers scored in a blind fashion manner in relation to the MeCP2 genotype, drug treatment, and age of the animal.

The bar cross test, as previously described (Ferrer et al, 2005; Lopez-Erauskin et al, 2011), was also carried out. In brief, it was done using a $100-\mathrm{cm}$-long and 2-cm-wide wooden bar that was wide enough for mice to stand on with their hind feet hanging over the edge, such that any slight lateral misstep would result in a slip. The bar was raised $50 \mathrm{~cm}$ above the bench surface so that animals did not jump off and would not be injured upon falling from the bar. The mice were placed at one end of the bar and were expected to cross to the other end. To eliminate the novelty of the task as a source of slips, all animals underwent three trials on the bar the day before and at the beginning of the testing session. In an experimental session, the numbers of hindlimb lateral slips and falls from the bar were counted in three consecutive trials. If an animal fell, it was placed back on the bar at the point from which it had fallen, and was allowed to complete the task.

\section{Western Blot}

For western blotting, animals were killed 4 weeks after the first treatment (at 8 weeks of age), and the MB area with the dopaminergic region was rapidly removed from the brain and cut with a Rodent Brain Slicer matrix (Zivic Instruments; $2 \mathrm{~mm}$ sections). Next, the protein extract was obtained with lysis buffer ( $20 \mathrm{mM}$ Tris-HCl, $\mathrm{pH} 7.5,150 \mathrm{mM}$ $\mathrm{NaCl}, 2 \mathrm{mM}$ EGTA, $0.1 \%$ Triton X-100, and complete protease inhibitor and phosphatase inhibitor cocktail tablet from Sigma and Roche) sonicated and denatured for $10 \mathrm{~min}$ at $95^{\circ} \mathrm{C}$. Protein concentrations were determined using the BCA (Pierce BCA Protein Assay kit). An amount of $25 \mu \mathrm{g}$ of each protein sample was separated on a $10 \%$ SDS-polyacrylamide gel by sodium dodecyl sulfate electrophoresis, and transferred onto a PVDF membrane (Immobilon-P, Millipore) by liquid electroblotting (Mini Trans-Blot Cell, Bio-Rad) for $1 \mathrm{~h}$ at $100 \mathrm{~V}$. The membrane was blocked in $5 \%$ nonfat dry milk in TBS $0.1 \%$ Tween 20 for $1 \mathrm{~h}$ at room temperature (RT). Primary antibody specific to Th (Millipore) was used at a dilution of 1:1000, overnight (ON) at $4{ }^{\circ} \mathrm{C}$. After extensive washing, the membrane was incubated with secondary antibody peroxidase-conjugate for $1 \mathrm{~h}$ at RT. Finally, the specific reaction was detected with a chemiluminescence reagent kit (Supersignal West Pico chemiluminiscent, Thermo Scientific). Digital images were processed with a GS-800 Calibrated Densitometer (BioRad). Bands were quantified with Bio-Rad Quantity One software. In all cases, images correspond to the results of one representative experiment out of three. The level of Th was normalized with an antibody to actin, used as a loading control.

\section{Immunofluorescence Analysis}

Mice were deeply anesthetized with dolethal (Vetoquinol), perfused transcardially with $\mathrm{NaCl}(0.9 \%)$ followed by buffered $4 \%$ paraformaldehyde in saline-phosphate buffer, $\mathrm{pH}$ 7.5. Brains were post-fixed $\mathrm{ON}$ in the same solution, subsequently dehydrated in $30 \%$ sucrose until the contents reached the bottom of the tube. All brains were dissected into $2 \mathrm{~mm}$ coronal sections (Rodent Brain Slicer matrix, Zivic Instruments) and cryoprotected in OCT, then stored at $-80^{\circ} \mathrm{C}$. Sections of $25 \mu \mathrm{m}$ were cut from the MB region with SNpc using a cryostat (Microm Microtech). The brain regions were identified using AGEA Allen Brain Atlas Mouse. All the immunostaining steps were carried out freefloating in solution. The staining protocol followed was based on previous detailed descriptions (Roux et al, 2007; Dura et al, 2008). All tissues were permeabilized and blocked with $0.2 \%$ of Triton X-100, $20 \%$ goat serum/PBS for $1 \mathrm{~h}$ at RT, and incubated with the appropriate primary antibodies $(1: 1000$ mouse Th and $1: 1000$ rabbit phosphoTh; MS X Th, Millipore; and Phospho-Th, Cell Signaling) in $0.2 \%$ Triton $\mathrm{X}-100,2 \%$ goat serum/PBS ON at $4{ }^{\circ} \mathrm{C}$. Anti-mouse Alexa 488/anti-rabbit Alexa 555 were used as secondary antibodies. DAPI (4,6-diamino-2-phenolindol dihydrochloride) counterstaining was performed on all sections, in order to visualize the nucleus, and coverslips were mounted in Mowiol (Calbiochem) after staining. The double-labeled images were acquired using a Leica TCS SP5 Spectral Confocal microscope (Leica, Milton Keynes, UK; magnification: HCX PL APO lambda blue $\times 63$ oil, numerical aperture (NA): 1.4 , and $1024 \times 1024$ resolution; Acquisition Software: Leica Application suite Advanced Fluorescence, version 2.6.0.7266). $\mathrm{Z}$ project and Tile were carried out in order to achieve complete staining with both antibodies. Densitometric analysis of the staining level was performed on 8-bit images using ImageJ Fiji $v s \quad 1.47$ software (http://rsb.info.nih.gov). The integrated density was calculated as the sum of the pixel values in a region of interest. We obtained pictures from different slices in the same region and then calculated the average of the successive impaired sections for each animal in each experimental group (total five).

\section{Golgi Staining}

Golgi staining was done to study the neuronal morphologies (FD Rapid GolgiStain Kit, FD Neurotechnologies). Animals were anesthetized as described above. Brains were extracted and all protocols were performed according to the manufacturer's instructions. Three brains from each of the treated groups were dissected into 2-mm coronal pieces (Rodent Brain Slicer matrix, Zivic Instruments), mounted in freezing medium (TFM, Triangle Biomedical) and stored at $-80^{\circ} \mathrm{C}$. Sections of $80-100 \mu \mathrm{m}$ were cut using a cryostat and mounted on gelatin-coated microscope slides with solution C. We proceeded with the staining protocols by following the instructions included in the manual. Between 10 and 15 neurons of the hippocampus zone were analyzed from two animals from each group. Images of dendritic spines were acquired with a Zeiss Axio Observer Z1 with an Apotome microscope (magnification: Plan-APOCHROMAT; $\times 63$ oil Dic NA. 1.4; Acquisition Software: ZEN 2011), and reconstructed in three dimensions using the NeuronStudio software for image processing to enable higher identification and to improve the quality of the spine analysis (Rodriguez et al, 2006; Rodriguez et al, 2008). Dendritic 
spines were counted by Sholl analysis over a length of $20 \mu \mathrm{m}$ with concentric circles of $10 \mu \mathrm{m}$ (Sholl, 1953).

\section{RNA Isolation from MB and Real-Time qPCR Analysis}

For total RNA preparation, the tissue was resuspended in TriZol (Life Technologies), purified with chloroform and precipitated with isopropanol, then washed with ethanol, and dissolved in sterile water. cDNA synthesis was performed with $1 \mu \mathrm{g}$ total RNA and random hexamers primers using the superscript KIT (Life Technologies). Aliquots $(1 \mu \mathrm{l})$ of diluted cDNA $(1: 4)$ were amplified by the SYBR Green PCR Master Mix (Life Technologies) in a final volume of $10 \mu \mathrm{l}$. Real-time PCRs were performed in triplicate on an Applied Biosystems 7900HT Fast Real-Time PCR system. All primer pairs were designed with Primer 3 software and validated by gel electrophoresis to amplify specific single products. All data were normalized with respect to an endogenous control: PPIA or RPL38. Relative mRNA levels were calculated using qBASE plus software (Biogazelle). PCR cycles were divided into initial denaturation at $95^{\circ} \mathrm{C}$ for $5 \mathrm{~min}$, followed by 40 cycles of $95^{\circ} \mathrm{C}$, for $30 \mathrm{~s}, 60^{\circ} \mathrm{C}$ for $30 \mathrm{~s}$, and $72{ }^{\circ} \mathrm{C}$ for $30 \mathrm{~s}$. The following primers were used: Drd1 (forward 5'-ATGGCTCCTAACACTTCTACCA-3'; reverse $5^{\prime}$-GGGTATTCCCTAAGAGAGTGGAC- $3^{\prime}$ ), $\quad$ Drd2 (forward $5^{\prime}$-TGGATCCACTGAACCTGTCC-3'; reverse $5^{\prime}$-TT GTAGTGGGGCCTGTCTG-3'), PPIA (forward 5'-CAAATG CTGGACCAAACACAA-3'; reverse $5^{\prime}$-GTTCATGCCTTCTT TCACCTT-3'), RPL38 (forward 5'-AGGATGCCAAGTCTGT CAAGA-3'; reverse $5^{\prime}$-TCCTTGTCTGTGATAACCAGGG-3').

\section{Dopamine Assay}

For the determination of dopamine levels in brain regions, the Mouse Dopamine Elisa Kit was used (Blue Gene, cat. no. E03D0043, Shanghai, China). In brief, mice were killed at 8 weeks and the studied brain regions were dissected and snap-frozen. Samples were weighed and homogenized in presence of saline solution $(20 \mu \mathrm{l} / \mathrm{mg}$ tissue). The resulting suspension was then sonicated and centrifuged to remove insoluble material. The samples were diluted and assayed according to the manufacturer's protocol. The final dopamine concentration for each sample was normalized with the respective total protein amount.

\section{Glutathione Assay Kit}

For the determination of total glutathione (GSx), reduced glutathione (GSH), and oxidized (GSSG) a Glutathione Assay Kit (Bio Vision, cat. no. K264-100 kit; Milpitas, California) was used. At 8 weeks of age, the mice were killed, and the brain was rapidly removed. Brain tissues were washed in $0.9 \% \mathrm{NaCl}$ solution and homogenized on ice with $100 \mu \mathrm{l}$ ice-cold Glutathione Assay Buffer for $40 \mathrm{mg}$ of tissue. $60 \mu \mathrm{l}$ of each homogenate was placed into a tube containing $20 \mu \mathrm{l}$ of cold perchloric Acid (PCA) and vortexed several seconds to achieve a uniform emulsion. Next, the samples were centrifuged and the supernatant (containing GSH) was collected. Ice-cold $\mathrm{KOH}(20 \mu \mathrm{l})$ was added to $40 \mu \mathrm{l}$ of PCA preserved samples to precipitate the PCA and neutralize the samples. In order to detect the GSH, we add assay buffer, until $90 \mu \mathrm{l}$ final volume. To obtain the GSx,
$70 \mu \mathrm{l}$ of assay buffer was added to $10 \mu$ of each sample with $10 \mu \mathrm{l}$ of Reducing Agent Mix to convert GSSG to GSH. To get the results of GSSG, to the $60 \mu \mathrm{l}$ of assay buffer and $10 \mu \mathrm{l}$ of sample, $10 \mu \mathrm{l}$ of GSH Quencher was added to quench GSH. After $10 \mathrm{~min}$ of RT incubation, $10 \mu \mathrm{l}$ of Reducing Agent Mix was added to destroy the excess GSH Quencher and convert GSSG to GSH. After 10 min of RT incubation, $10 \mu \mathrm{l}$ of o-phthalaldehyde probe was added to all samples. Next, all samples were incubated at RT in the dark for at least $30 \mathrm{~min}$. The fluorescence was read at $\mathrm{Ex} / \mathrm{Em}=340$ / $420 \mathrm{~nm}$

\section{Statistical Analysis}

The data obtained from WT and KO animals in the initial experiments were compared using Student's unpaired $t$-test. Score data, bar cross, immunofluorescence, qPCR analysis, Golgi staining, and western blot experiments in the different treatments groups were compared by one-way analysis of variance (ANOVA) with Tukey's or Bonferroni's Multiple Comparison post hoc test for intergroup comparisons. All results were analyzed using GraphPad 5.04 Prism software. Life span data were plotted as Kaplan-Meier survival curves. Results were considered significant for values of ${ }^{*} p<0.05,{ }^{* *} p<0.01$, or ${ }^{* *} p<0.001$. Data are presented as the mean and SEM.

\section{RESULTS}

The Combined L-Dopa + Ddci Treatment of Mecp2 Null Mice is Well Tolerated, Diminishes RTT-Associated Symptoms, and Increases Life span

RTT patients and Mecp2 KO mice manifest a progressive postnatal neurological phenotype (Guy et al, 2001; Chahrour and Zoghbi, 2007). Mecp2 KO mice are apparently normal until 4 weeks of age and then begin to suffer cognitive and motor dysfunctions. The progression of symptoms in the Mecp2 KO male mice leads to rapid weight loss and death at $\sim 10$ weeks of age in comparison with WT animals (Guy et al, 2001). To test the effect of the L-Dopa + Ddci combination on the symptomatology of RTT mice, we treated the animals daily over 6 weeks. We used a once-a-day dose of intraperitoneal Ddci, L-Dopa or L-Dopa + Ddci. In the L-Dopa + Ddci group, the Ddci drug was injected $15 \mathrm{~min}$ before L-Dopa administration. The used doses are those that have proven efficient in mice models of Parkinson's disease (Chartoff et al, 2001; Santini et al 2009). Every 2 days, we scored the neurological recovery of the MeCP2 model mice as previously described (Guy et al, 2007). We started the treatments when the animals were 4 weeks old because by 6 weeks of age it is already possible to observe some of the characteristic symptoms of the Mecp2 KO model, that is, reduced mobility, retraction of the legs, tremor, irregular breathing, and difficulties with walking. First, we determined whether the administration of L-Dopa, Ddci, or the combination L-Dopa + Ddci was toxic for the Mecp2 KO mice by daily monitoring the weight of each animal. The Mecp2 KO mice treated with either the vehicle (saline serum), L-Dopa, Ddci, or the combination L-DOPA + Ddci showed had similar weights, independent of the compound used (Figure 1a). At the time of killing, 

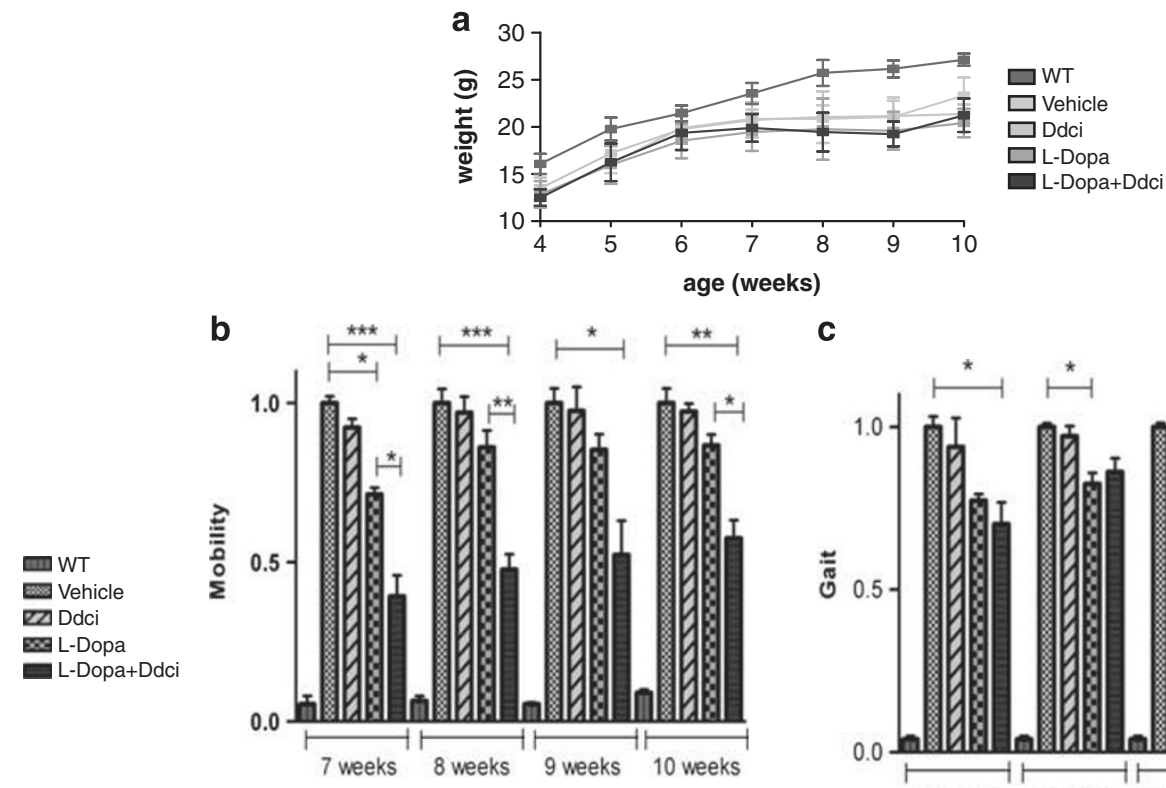

\section{C}
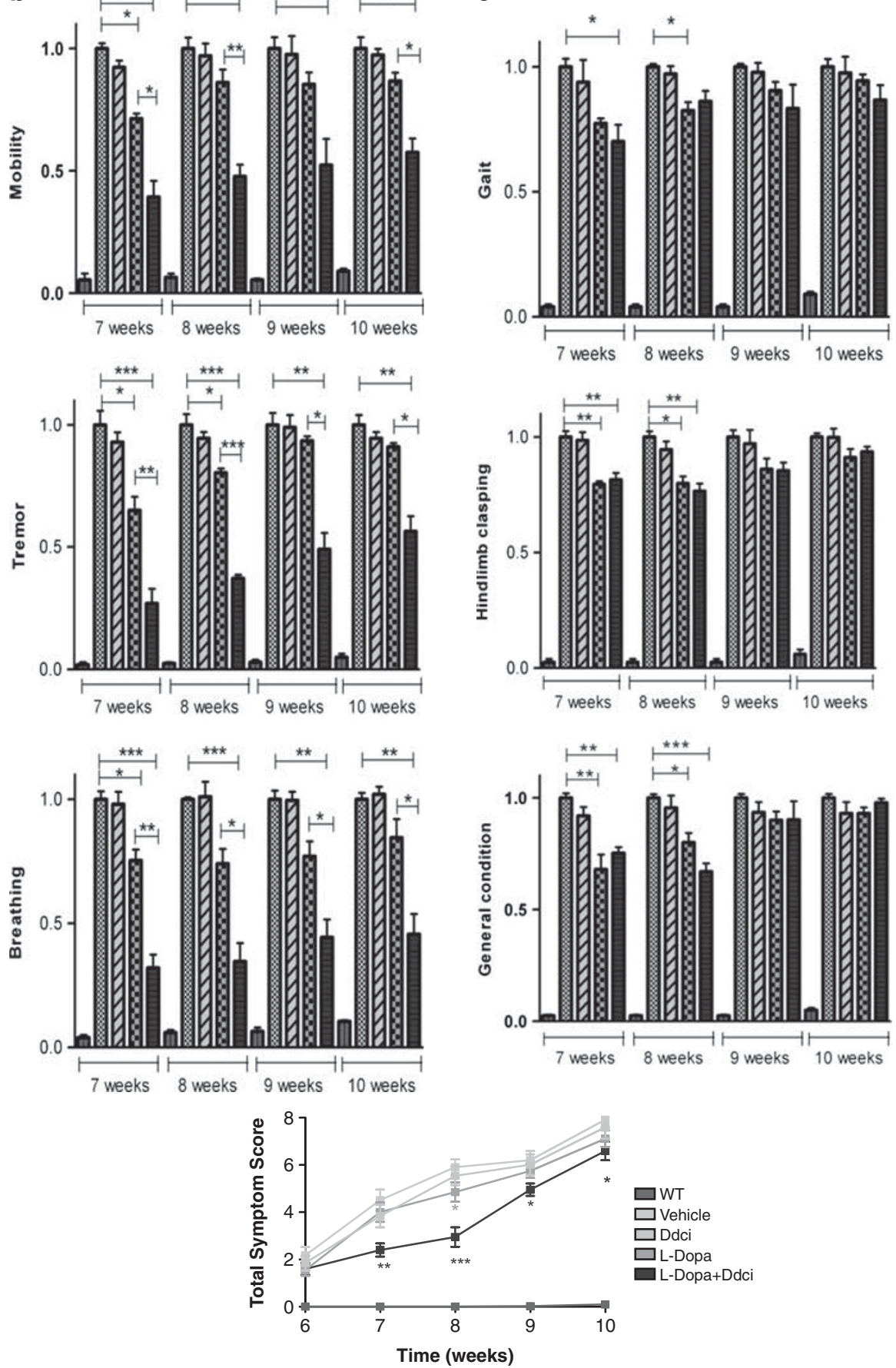

Figure I Effect of L-Dopa and L-Dopa + Ddci (dopa-decarboxylase inhibitor) treatments on the Mecp2 null phenotype. The number of animals used in each experiment was 30 for wild-type (WT) control, 37 for vehicle, 40 for L-Dopa + Ddci, 30 for L-Dopa, and I 5 for Ddci. (a) Measurement of mice body weight during treatment. No effect on body weight was observed after the treatments in the Mecp2 KO mice. (b) Plots showing 'Score Test' (Guy et al, 200I) improvement upon L-Dopa + Ddci treatment related to mobility, tremor, and breathing. (c) Plots showing 'Score Test' (Guy et al, 200I) improvement related to gait, hindlimb clasping, and general conditions that are less affected by the treatments. (d) Plot of total average symptom scores showing 'Score Test' (Guy et al, 200 I) improvement in Mecp2 KO-treated mice upon L-Dopa + Ddci treatment. For the described pairwise comparisons, a Tukey HSD post hoc test was performed after the one-way analysis of variance. ${ }^{*} p<0.05$, $* * p<0.01$, and $* * * p<0.001$. 
liver tissues were resected for pathological analysis, and no toxicity was detected in any of the mice used in the different drug treatments (Supplementary Figure 1). Finally, we have studied possible neutoxicity by measuring oxidative stress in the brain of these animals. The ratio of reduced (GSH) vs oxidized (GSSG) GSH shows that the Mecp2 KO mice undergoes a significant oxidative stress in comparison with the WT littermate mice (Supplementary Figure 1), and that the L-Dopa + Ddci treatment significantly reduces oxidative stress in the brain of these animals (Supplementary Figure 1).

We next analyzed the effect of the L-Dopa+Ddci combined treatment in the Mecp2 KO mice on the clinicopathological phenotype of this RTT model. We measured score mobility, tremor, breathing, gait, hindlimb clasping, and general condition (Guy et al, 2001; Guy et al, 2007) (Figure $1 \mathrm{~b}$ and $\mathrm{c}$ ). The plots show the overall distribution of symptoms at 7, 8, 9, and 10 weeks of age, and compare the scores of the vehicle, L-Dopa, Ddci, and L-Dopa + Ddci-treated groups. During each week of treatment, the 'Score Test' values (Guy et al, 2001) were separately calculated, then normalized with respect to the vehicle group using one-way ANOVA with Bonferroni's post hoc multiple comparison. We confirmed the existence of a significant improvement in mobility, tremor, and breathing from 7 to 10 weeks of age in the vehicle $v s$ L-Dopa group as has been previously described (Panayotis et al, 2011), but remarkably the improvement in the symptoms was significantly greater in the L-Dopa + Ddci-treated group, $p<0.001$ (Figure 1b). There was no difference between vehicle and Ddci alone (Figure 1b). Treatment with L-Dopa + Ddci improved the mobility, tremor, and breathing phenotype by an average of $50 \%$, having less effect on older mice. Regarding the phenotypes of gait, hindlimb clasping or general condition symptom, a significant improvement was observed with both L-Dopa and L-Dopa + Ddci treatments in younger mice (7-8 weeks) that became a nonsignificant trend in older mice (9-10 weeks; Figure 1c). If we plot the average of the eight scored symptoms to create a total score (Guy et al, 2001), a significant phenotypic improvement is observed in the L-Dopa+Ddci-treated group (Figure 1d). We further determined the possible restoration of the motor impairments in the Mecp2 null mice upon administration of L-Dopa + Ddci therapy by using the bar cross test (Ferrer et al, 2005; Lopez-Erauskin et al, 2011). We observed that L-Dopa alone was able to improve test performance in comparison with the vehicletreated group, but the Rett model mice receiving the combined L-Dopa + Ddci regimen achieved the best scores, such as a shorter time to cross the bar (Figure 2a) and fewer slips (Figure 2b). Illustrative movies of the bar cross test in the WT animal and in the Mecp2 null mice treated with the vehicle or the L-Dopa + Ddci combination are included in Supplementary Material. Overall for the tests used, the efficacy of the studied combination was particularly evident in the first weeks when there may be a window for therapeutic opportunities. Most importantly, the observed improvement in the disease-linked features was also associated with an increased life span of the male Mecp2 null mice that received the L-Dopa + Ddci combination therapy ( $p<0.001$, Kaplan-Meier log-rank test; Figure 2e).

Thus, the combined administration of L-Dopa + Ddci in Mecp2-deficient mice increases their well-being overall by diminishing RTT symptomatology, particularly those features controlled by the dopaminergic pathway in the nigrostratium, such as mobility, tremor, and breathing.

\section{The use of L-Dopa + Ddci in the Mecp2 Null Mice Induces Dendritic Growth Mediated by Dopaminergic Neurons}

Beyond the amelioration of the RTT clinical phenotype described above, we wondered whether the L-Dopa + Ddci treatment was also associated with an improvement in the pathological cellular and tissular changes observed in the brain of Mecp2 null mice. Morphological studies in postmortem brain samples from RTT individuals exhibited a characteristic neuropathology that included decreased
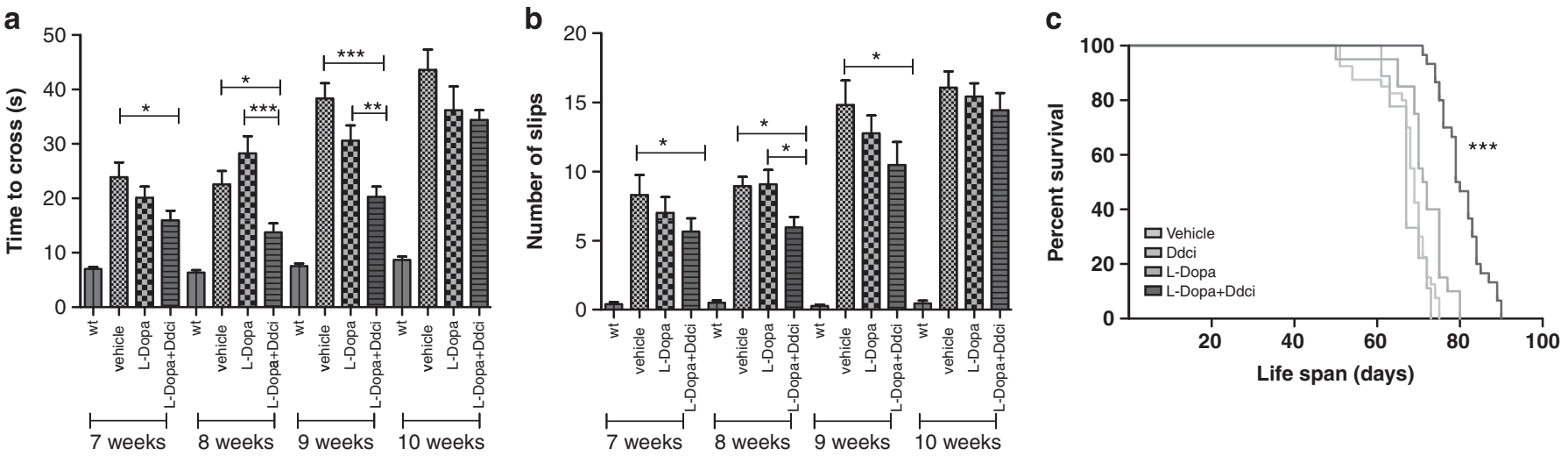

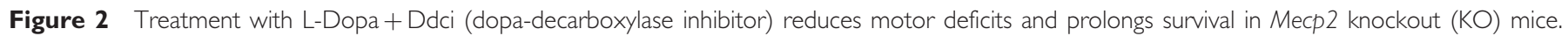

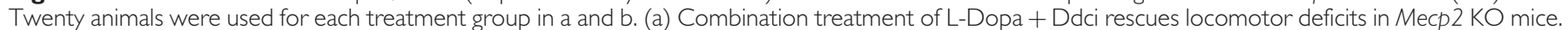

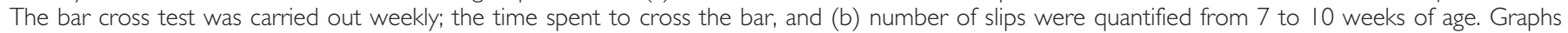
illustrate the mean and SEM of two independent experiments. For the described pairwise comparisons, a Tukey HSD post hoc test was performed after the

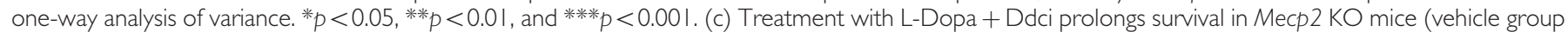

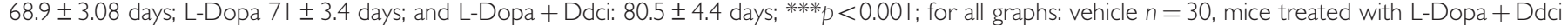
$n=30$, and mice treated with L-Dopa alone with $n=10$, Ddci group $n=10$ ). 

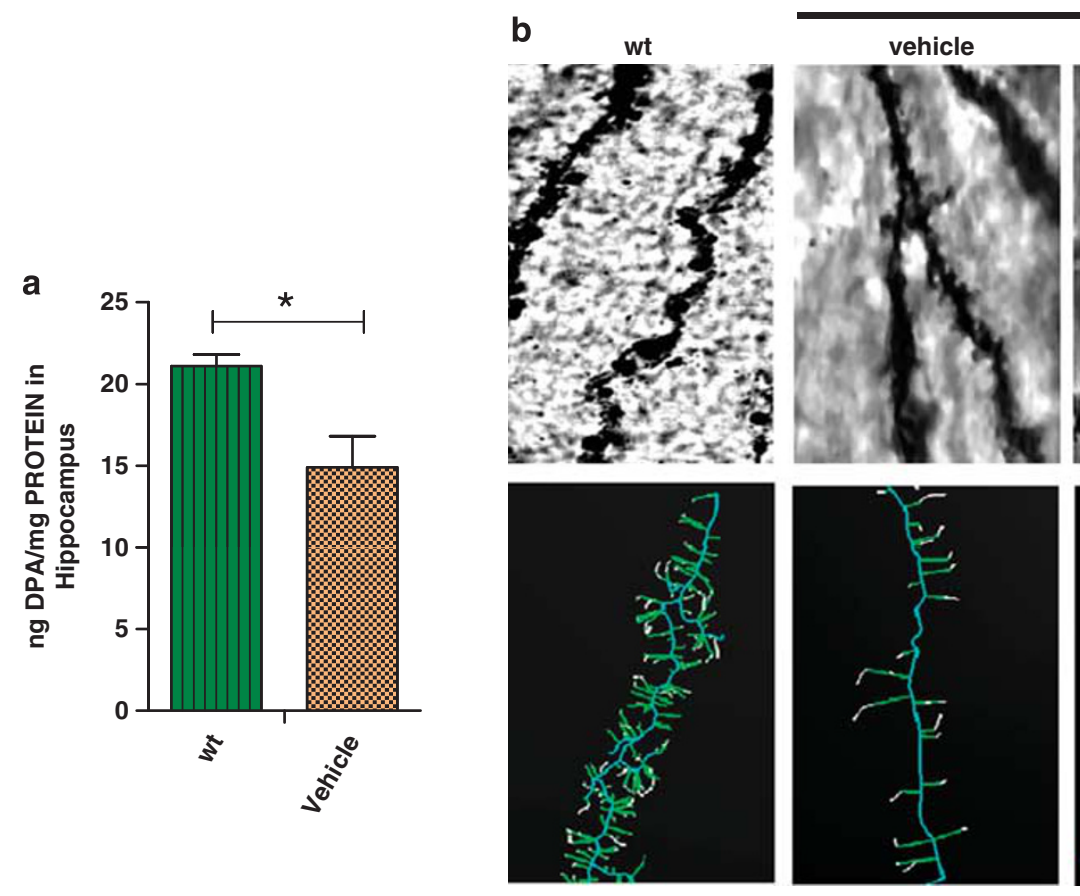

Mecp2 null mice
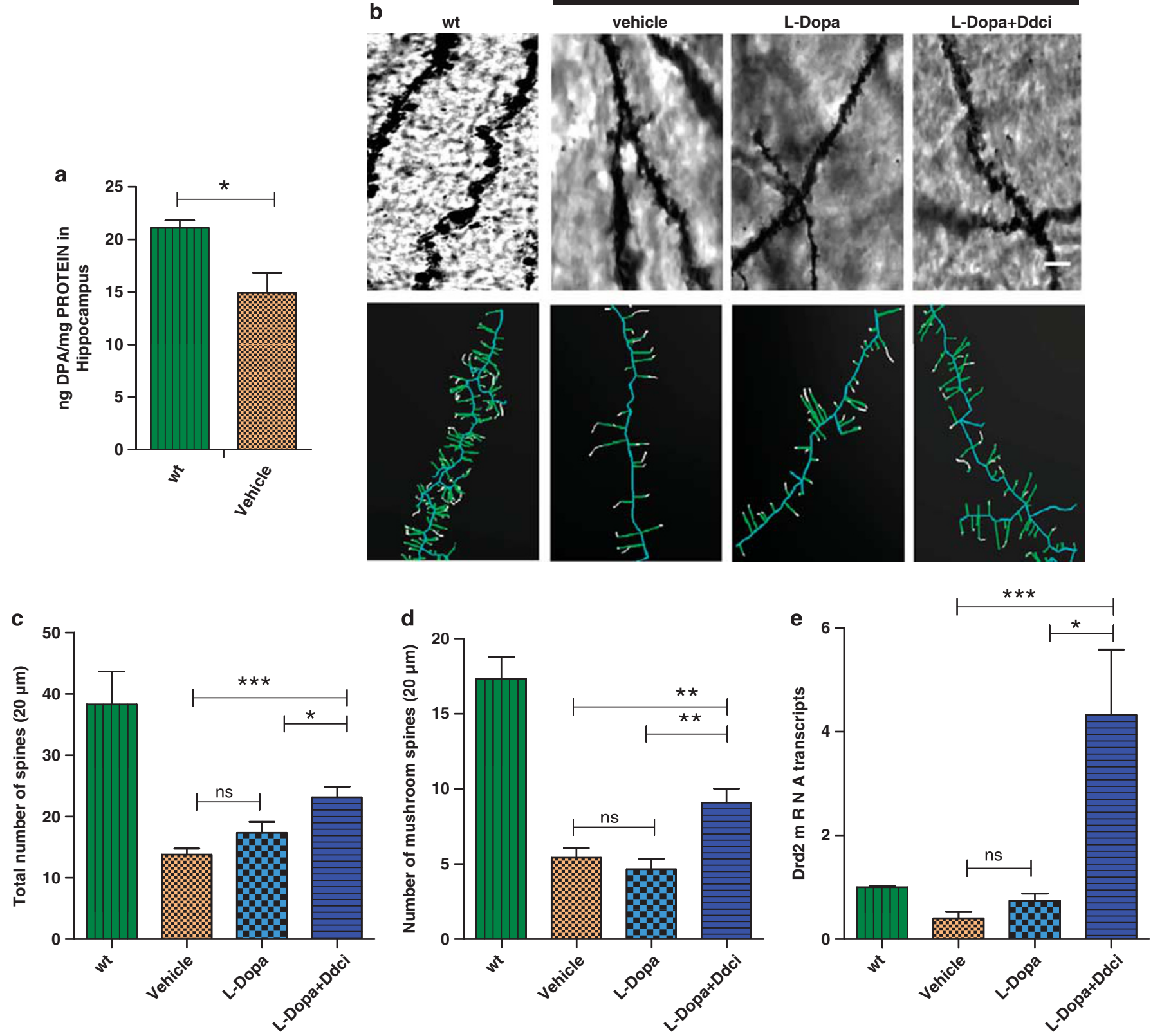

Figure 3 Determination of neuronal dendritic growth in the Mecp2 null mice upon L-Dopa + Ddci (dopa-decarboxylase inhibitor) treatment. (a) Dopamine concentration normalized with the respective total protein amount in hippocampus of wild-type (WT) and Mecp2 null mice. (b) Spine density in hippocampus of WT and Mecp2 null mice with or without treatment acquired with a Zeiss wide-field microscope, $\times 63$ magnification, I.4 numerical aperture. (Below) Bars showing the spine density (mean and SEM) in the hippocampus region of Mecp2-deficient mice vs WT, and treated with L-Dopa + Ddci or L-Dopa alone, at 4 weeks post treatment (8 weeks of age), focusing on the total number of spines (c) and mushroom spines (d). Dendritic segments of $20 \mu \mathrm{m}$ were analyzed for each group, and images were obtained with a Zeiss microscope, $\times 63$ magnification, and processed with NeuronStudio software. The bars illustrate the average total count of spines in dendritic segments in the four experimental groups (Sholl analysis). (e) L-Dopa + Ddci treatment causes an increase in dopamine 2 receptor (D2R) levels. All transcript levels were determined using qRT-PCR (Applied Biosystem). The values (mean and SEM) are the ratio with respect to WT, which is assigned a value of I (line) * $p<0.05$, *** $<<0.01$. For the described pairwise comparisons, a Tukey HSD post hoc test was performed after the one-way analysis of variance. $* p<0.05$, ** $p<0.01$, and $* * * p<0.001$.

neuronal size and increased neuronal density in the cerebral cortex, hypothalamus and the hippocampal formation (Bauman et al, 1995), and decreased dendritic growth in pyramidal neurons of the subiculum and frontal and motor cortices (Armstrong et al, 1995). The quantitative analyses of dendritic spine density in postmortem brain tissue from
RTT individuals also revealed that hippocampal CA1 pyramidal neurons have lower spine density than agematched female control individuals (Chapleau et al, 2009). In the Mecp2 KO mice, CA1 pyramidal neurons showed lower dendritic spine density than those from WT littermates (Chapleau et al, 2012). These observations are 

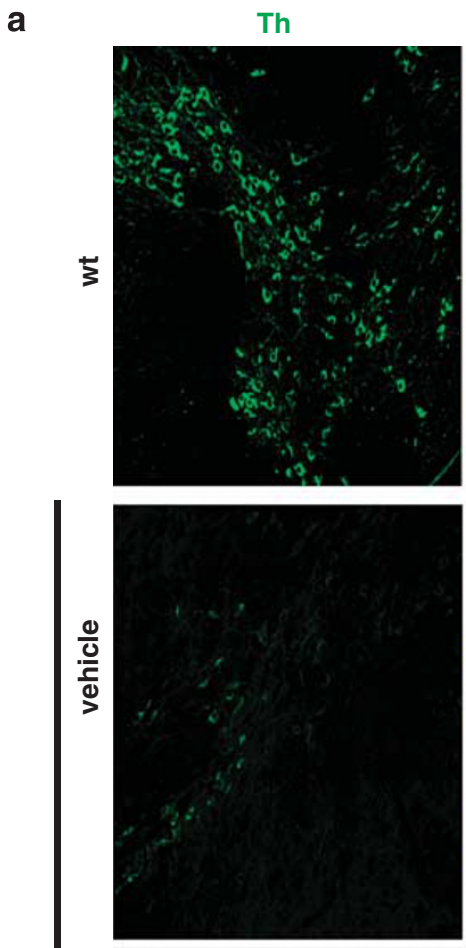

를
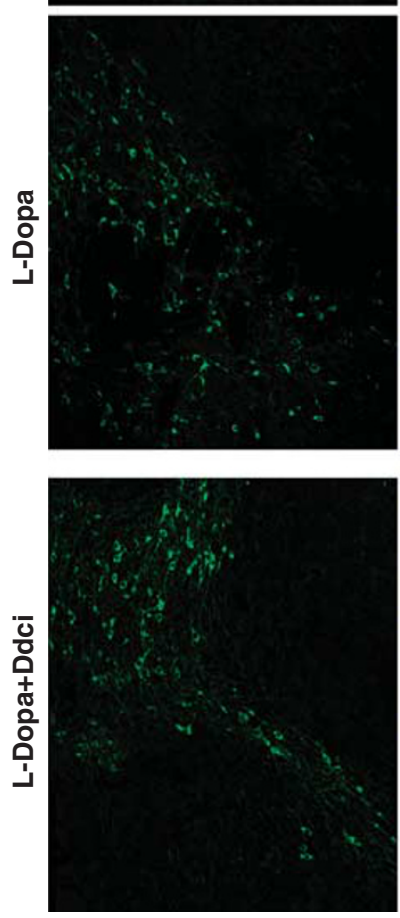

Dapi
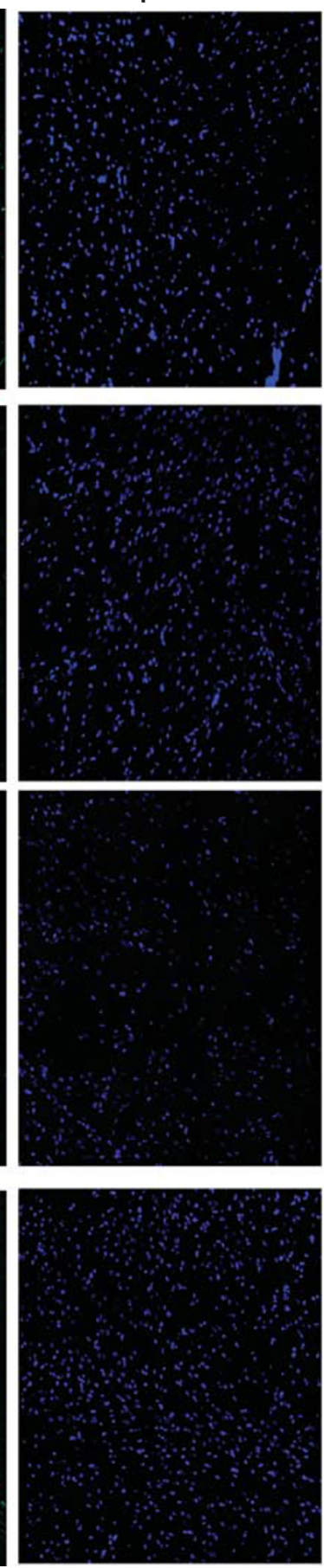

Merge Th
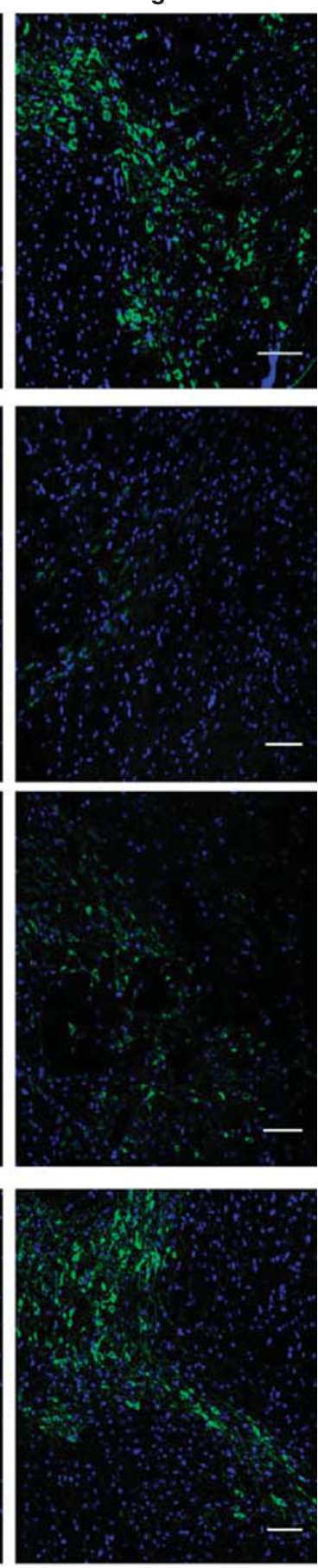

b

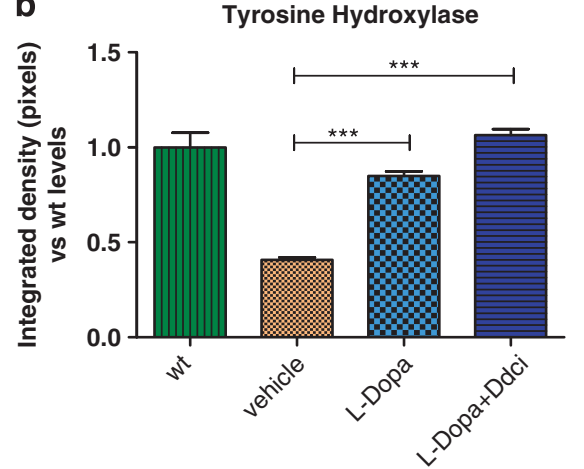

C
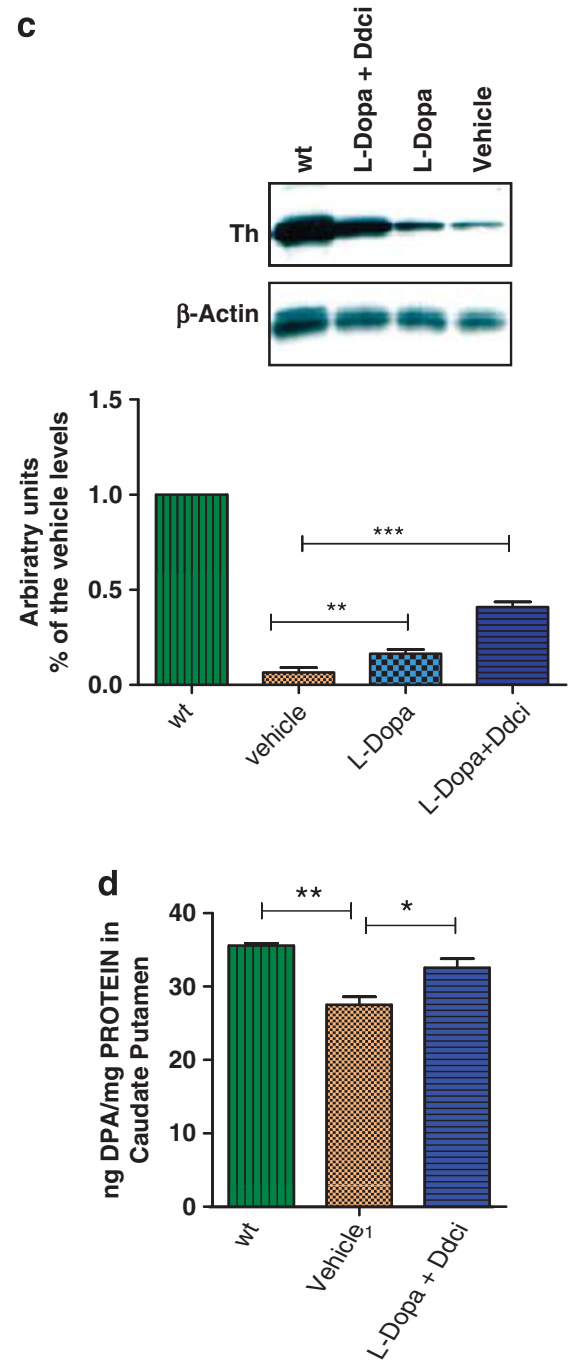

Figure 4 Determination of tyrosine hydroxylase (Th) expression in the Mecp2 null mice upon different treatments. (a) Representative immunostaining of coronal brain sections from Substantia nigra pars compacta (SNpc). Midbrain dopaminergic neurons were coimmunostained for Th (green) and DAPI (4,6diamino-2-phenolindol dihydrochloride) counterstain (blue). Scale bars, I00 $\mu \mathrm{m}$. (b) Quantification of Th-positive staining in all aforementioned experimental groups. Bars represent the integrated density (Image) Fiji vs, 1.47) of the picture obtained with a SPS5 confocal microscope, $\times 63$ objective. An increase in Th levels in Mecp2-deficient mice treated with L-Dopa and L-Dopa + Ddci is observed. (c) Representative western blot showing the levels of Th proteins in midbrain from four experimental groups. Actin was used as a loading control. (d) Dopamine concentration normalized with the respective total protein amount in caudade putamen of wild-type (WT) and Mecp2 null mice treated with vehicle or L-Dopa + Ddci (dopa-decarboxylase inhibitor). All animals were analyzed 4 weeks post treatment ( 8 weeks old), $n=10$ per group. One-way analysis of variance (ANOVA) and a post hoc test were used for statistical comparison. For the described pairwise comparisons, a Tukey HSD post hoc test was performed after the one-way ANOVA. * $p<0.05$, *** $<<0.01$, and ***** $p<0.001$. 
particularly important in our model because Dopamine facilitates dendritic spine formation, and the improvement of the symptomatology in the studied Mecp null mouse model upon L-Dopa + Ddci use could be in part explained by the stimulation of neuronal dendritic growth. In this regard, we found that the hippocampus of Mecp2 $\mathrm{KO}$ mice compared with control littermates showed significant lower levels of dopamine (Figure 3a). Because most of the abnormalities in morphology, density of dendritic spines, and reduction of synaptic activity in RTT occur in the hippocampus region (Fiala et al, 2002; Dani et al, 2005; Chang et al, 2006; Chao et al, 2007), we evaluated the dendrite status in this region in the differently treated Mecp2 null mice.

Using Golgi staining (Figure 3b) and NeuronStudio software and the Sholl analysis algorithm (Rodriguez et al, 2008), we found that the RTT mice treated with the L-Dopa + Ddci combination showed a significant increase in the number of spines in comparison with vehicle-treated animals (Figure 3c). Further refinement of the analyses by counting mushroom spine density, as an example of the more mature type of spines (Chapleau et al, 2009), also showed a significant increase of this class of spines in the Mecp2 null mice that received the L-Dopa + Ddci treatment (Figure 3d). Most importantly, the increase in spinogenesis under the combined therapy regimen is associated with the enhanced expression of the Dopamine 2 Receptor (D2R) in the $\mathrm{MB}$ of the Rett mouse model (Figure 3e), with an additional beneficial amplification effect for the L-Dopa + Ddci treatment because D2R elicits extensive spine formation (Fasano et al, 2013). The role of Dopamine in reverting Mecp2 null mice features is also highlighted by the study of the expression levels of Th, the rate-limiting enzyme of dopamine synthesis, which works by catalyzing the hydroxylation of tyrosine to L-Dopa (Molinoff and Axelrod, 1971). The Mecp2 null mice exhibit low levels of Th expression in comparison with the WT group, assessed by immunofluorescence staining in $\mathrm{MB}$ (Figure $4 \mathrm{a}$ and $\mathrm{b}$ ), and we observed a recovery of physiological levels of Th in the L-Dopa + Ddci-treated group. These results are consistent with the increase of Th-expressing neurons observed upon L-Dopa in Parkinson disease patients (Porrit et al, 2000), animal models of Parkinson disease (Betrabet et al, 1997; Tandé et al, 2006; Espadas et al, 2012) and, most importantly, in the studied RTT mouse model (Panayotis et al, 2011; Kao et al, 2013). Herein, the addition of Ddci to the L-Dopa treatment further enhance Th expression (Figure $4 \mathrm{a}$ and $\mathrm{b}$ ). Western blot analyses confirmed the upregulation of Th in the Mecp2 null group that received the combined treatment (Figure 4c). Furthermore, the L-Dopa + Ddci-treated group significantly recovered dopamine levels in comparison with the vehicle-treated group (Figure 4d). Thus, the formation of new spines induced by an activation of dopaminergic neurons is a likely mechanism that explains the observed improvement of the RTT phenotype upon the combined administration of L-Dopa + Ddci.

\section{DISCUSSION}

Overall, our results indicate that the use of strategies similar to those used in the therapy of Parkinson disease could also be useful for treating RTT. Herein, we show that the combination of L-Dopa and the peripherally acting aminoacid decarboxylase inhibitor benserazide, a commonly used therapeutic regimen in Parkinson diseases in the formulation known as Madopar, can improve RTT symptomatology in association with the stimulation of neuronal dendritic growth. These results link with the dopaminergic defects observed in RTT, such as the well-known reduction in levels of dopamine in the brain and cerebrospinal fluid analyses (Riederer et al, 1986; Lekman et al, 1989; Wenk et al, 1991), a low D2R content (Chiron et al, 1993), and reduced Th immunoreactivity (Jellinger et al, 1988). In the RTT mouse models, the dopamine levels are also reduced (Ide et al, $2005)$ and there is a deficiency of Th-expressing neurons (Viemari et al, 2005). This dopamine-associated impairment is associated, as in our study, with an abnormal thinning of dendrites (Jellinger, 2003), and dendritic spine density is also significantly diminished in Mecp2 KO mice (Nguyen et al, 2012). Most importantly, the results extend the original observation that L-Dopa treatment ameliorated the motor deficiency of the RTT mouse model (Panayotis et al, 2011) to formally prove that the addition of the Ddci is able to further improve the clinical symptoms of the disease with consequences at the molecular and cellular levels in the brains of these animals. In comparison with the pioneering study of Panayotis et al (2011), we used a higher dose of L-Dopa, an intraperitoneal injection instead of an oral administration, and the effects were more obvious in younger ages. Importantly, both studies support that a targeting of the dopamine pathway improves the Rett phenotype in the described mice model. However, a caution note should also be included because we observed that the improvement of the phenotype diminished with the duration of the treatment and our survival curves did not extend beyond 90 days.

To our knowledge, the biomedical literature only features a single case of a Rett patient who has received L-Dopa, but the authors did not study its effect on the patient's mobility, tremor, and breathing (Nomura et al, 1997). However, we know that the combination of L-Dopa and another Ddci (carbidopa) is able to improve the symptomatology of Th-deficient patients (Segawa syndrome, OMIM 606407) (Ludecke et al, 1995; Dionisi-Vici et al, 2000). These results are very interesting because we confirmed the diminished expression of Th in the RTT mouse model (Panayotis et al, 2011; Kao et al, 2013) and because the Segawa syndrome features some clinical overlap with RTT, being a progressive neurodevelopmental disorder with onset during the first year of life, Parkinsonian features and respiratory distress (Ludecke et al, 1995; Brautigam et al, 1999).

Overall, the findings reported herein are promising to overcome the dopaminergic defects observed in this preclinical model of RTT, but much effort will be required to extend the duration of the benefits of the described treatments or to analyze its effect in female mice in future studies of this devastating disorder.

\section{FUNDING AND DISCLOSURE}

The authors declare no conflict of interest. 


\section{ACKNOWLEDGEMENTS}

This study was supported by the European Community's Seventh Framework Program (FP7/2007-2013), under grant agreement PITN-GA-2012-316758- EPITRAIN project and PITN-GA-2009-238242-DISCHROM; ERC grant agreement 268626-EPINORC project; the E-RARE EuroRETT network (PI071327) and the CIBER on Rare Diseases (CIBERER) from the Carlos III Health Institute); the Fondation Lejeune (France); MINECO projects SAF2011-22803, CSD2006-00049; the Cellex Foundation; the Botín Foundation; the Catalan Association for Rett Syndrome; and the Health and Science Departments of the Catalan Government (Generalitat de Catalunya) projects AGAUR 2009SGR1315 and 2009SGR85. KS and PP are EPITRAIN Research Fellows; SF is a Miguel Servet Researcher (CP11/00080); and ME and AP are ICREA Research Professors.

\section{REFERENCES}

Amir RE, Van den Veyver IB, Wan M, Tran CQ, Francke U, Zoghbi HY (1999). Rett syndrome is caused by mutations in X-linked MECP2, encoding methyl-CpG-binding protein 2. Nat Genet 23: 185-188.

Armstrong D, Dunn JK, Antalffy B, Trivedi R (1995). Selective dendritic alterations in the cortex of Rett syndrome. J Neuropathol Exp Neurol 54: 195-201.

Armstrong D (1995). The neuropathology of Rett syndromeoverview 1994. Neuropediatrics 26: 100-104.

Bauman ML, Kemper TL, Arin DM (1995). Pervasive neuroanatomic abnormalities of the brain in three cases of Rett's syndrome. Neurology 45: 1581-1586.

Betarbet R, Turner R, Chockkan V, DeLong MR, Allers KA, Waltersm J et al (1997). Dopaminergic neurons intrinsic to the primate striatum. J Neurosci 17: 6761-6768.

Blandini F, Nappi G, Tassorelli C, Martignoni E (2000). Functional changes of the basal ganglia circuitry in Parkinson's disease. Prog Neurobiol 62: 63-88.

Brautigam C, Steenbergen-Spanjers GC, Hoffmann GF, DionisiVici C, van den Heuvel LP, Smeitink JA et al (1999). Biochemical and molecular genetic characteristics of the severe form of tyrosine hydroxylase deficiency. Clin Chem 45: 2073-2078.

Chahrour M, Zoghbi HY (2007). The story of Rett syndrome: from clinic to neurobiology. Neuron 56: 422-437.

Chang Q, Khare G, Dani V, Nelson S, Jaenisch R (2006). The disease progression of Mecp2 mutant mice is affected by the level of BDNF expression. Neuron 49: 341-348.

Chao HT, Zoghbi HY, Rosenmund C (2007). MeCP2 controls excitatory synaptic strength by regulating glutamatergic synapse number. Neuron 56: 58-65.

Chapleau CA, Boggio EM, Calfa G, Percy AK, Giustetto M, PozzoMiller L (2012). Hippocampal CA1 pyramidal neurons of Mecp2 mutant mice show a dendritic spine phenotype only in the presymptomatic stage. Neural Plast, published online 30 July 2012, doi:10.1155/2012/976164.

Chapleau CA, Calfa GD, Lane MC, Albertson AJ, Larimore JL, Kudo S et al (2009). Dendritic spine pathologies in hippocampal pyramidal neurons from Rett syndrome brain and after expression of Rettassociated MECP2 mutations. Neurobiol Dis 35: 219-233.

Chartoff EH, Marck BT, Matsumoto AM, Dorsa DM, Palmiter RD (2001). Induction of stereotypy in dopamine-deficient mice requires striatal D1 receptor activation. Proc Natl Acad Sci USA 98: 10451-10456.

Chiron C, Bulteau C, Loc'h C, Raynaud C, Garreau B, Syrota A et al (1993). Dopaminergic D2 receptor SPECT imaging in Rett syndrome: increase of specific binding in striatum. J Nucl Med 34: $1717-1721$.
Dani VS, Chang Q, Maffei A, Turrigiano GG, Jaenisch R, Nelson SB (2005). Reduced cortical activity due to a shift in the balance between excitation and inhibition in a mouse model of Rett syndrome. Proc Natl Acad Sci USA 102: 12560-12565.

Dionisi-Vici C, Hoffmann GF, Leuzzi V, Hoffken H, Brautigam C, Rizzo C et al (2000). Tyrosine hydroxylase deficiency with severe clinical course: clinical and biochemical investigations and optimization of therapy. J Pediatr 136: 560-562.

Dura E, Villard L, Roux JC (2008). Expression of methyl CpG binding protein 2 (Mecp2) during the postnatal development of the mouse brainstem. Brain Res 1236: 176-184.

Espadas I, Darmopil S, Vergano-Vera E, Ortiz O, Oliva I, VicarioAbejon C et al (2012). L-DOPA-induced increase in THimmunoreactive striatal neurons in parkinsonian mice: insights into regulation and function. Neurobiol Dis 48: 271-281.

Fasano C, Bourque MJ, Lapointe G, Leo D, Thibault D, Haber $M$ et al (2013). Dopamine facilitates dendritic spine formation by cultured striatal medium spiny neurons through both D1 and D2 dopamine receptors. Neuropharmacology 67: 432-443.

Ferrer I, Kapfhammer JP, Hindelang C, Kemp S, Troffer-Charlier N, Broccoli $\mathrm{V}$ et al (2005). Inactivation of the peroxisomal ABCD2 transporter in the mouse leads to late-onset ataxia involving mitochondria, Golgi and endoplasmic reticulum damage. Hum Mol Genet 14: 3565-3577.

Fiala JC, Spacek J, Harris KM (2002). Dendritic spine pathology: cause or consequence of neurological disorders? Brain Res Rev 39: 29-54.

FitzGerald PM, Jankovic J, Glaze DG, Schultz R, Percy AK (1990). Extrapyramidal involvement in Rett's syndrome. Neurology 40: 293-295.

Gamer M, Lemon J, Fellows I, Singh P (2012). irr: Various Coefficients of Interrater Reliability and Agreement. R package version 0.84 .

Guy J, Gan J, Selfridge J, Cobb S, Bird A (2007). Reversal of neurological defects in a mouse model of Rett syndrome. Science 315: 1143-1147.

Guy J, Hendrich B, Holmes M, Martin JE, Bird A (2001). A mouse Mecp2-null mutation causes neurological symptoms that mimic Rett syndrome. Nat Genet 27: 322-326.

Ide S, Itoh M, Goto Y (2005). Defect in normal developmental increase of the brain biogenic amine concentrations in the mecp2-null mouse. Neurosci Lett 386: 14-17.

Jellinger K, Armstrong D, Zoghbi HY, Percy AK (1988). Neuropathology of Rett syndrome. Acta Neuropathol 76: 142-158.

Jellinger KA (2003). Rett Syndrome - an update. J Neural Transm 110: $681-701$.

Jenner P (2008). Functional models of Parkinson's disease: a valuable tool in the development of novel therapies. Ann Neurol 64(Suppl 2): S16-S29.

Kao FC, Su SH, Carlson GC, Liao W (2013). MeCP2-mediated alterations of striatal features accompany psychomotor deficits in a mouse model of Rett syndrome. Brain Struct Funct (e-pub ahead of print 12 November 2013).

Kishi N, Macklis JD (2004). MECP2 is progressively expressed in post-migratory neurons and is involved in neuronal maturation rather than cell fate decisions. Mol Cell Neurosci 27: 306-321.

Kitt CA, Wilcox BJ (1995). Preliminary evidence for neurodegenerative changes in the substantia nigra of Rett syndrome. Neuropediatrics 26: 114-118.

Lekman A, Witt-Engerstrom I, Gottfries J, Hagberg BA, Percy AK, Svennerholm L (1989). Rett syndrome: biogenic amines and metabolites in postmortem brain. Pediatr Neurol 5: 357-362.

Lopez-Erauskin J, Fourcade S, Galino J, Ruiz M, Schluter A, Naudi A et al (2011). Antioxidants halt axonal degeneration in a mouse model of X-adrenoleukodystrophy. Ann Neurol 70: 84-92.

Ludecke B, Dworniczak B, Bartholome K (1995). A point mutation in the tyrosine hydroxylase gene associated with Segawa's syndrome. Hum Genet 95: 123-125. 
Luikenhuis S, Giacometti E, Beard CF, Jaenisch R (2004). Expression of MeCP2 in postmitotic neurons rescues Rett syndrome in mice. Proc Natl Acad Sci USA 101: 6033-6038.

Mena MA, Casarejos MJ, Solano RM, de Yebenes JG (2009). Half a century of L-DOPA. Curr Top Med Chem 9: 880-893.

Molinoff PB, Axelrod J (1971). Biochemistry of catecholamines. Annu Rev Biochem 40: 465-500.

Neul JL, Zoghbi HY (2004). Rett syndrome: a prototypical neurodevelopmental disorder. Neuroscientist 10: 118-128.

Nguyen MV, Du F, Felice CA, Shan X, Nigam A, Mandel G et al (2012). MeCP2 is critical for maintaining mature neuronal networks and global brain anatomy during late stages of postnatal brain development and in the mature adult brain. J Neurosci 32: 10021-10034.

Nomura Y, Kimura K, Arai H, Segawa M (1997). Involvement of the autonomic nervous system in the pathophysiology of Rett syndrome. Eur Child Adolesc Psychiatry 6: 42-46.

Olanow CW, Watts RL, Koller WC (2001). An algorithm (decision tree) for the management of Parkinson's disease (2001): treatment guidelines. Neurology 56(Suppl 5): S1-S88.

Panayotis N, Pratte M, Borges-Correia A, Ghata A, Villard L, Roux JC (2011). Morphological and functional alterations in the substantia nigra pars compacta of the Mecp2-null mouse. Neurobiol Dis 41: 385-397.

Porritt MJ, Batchelor PE, Hughes AJ, Kalnins R, Donnan GA, Howells DW (2000). New dopaminergic neurons in Parkinson's disease striatum. Lancet 356: 44-45.

$\mathrm{R}$ Core Team (2013). R: A Language and Environment for Statistical Computing. R Foundation for Statistical Computing, Vienna, Austria. ISBN 3-900051-07-0, http://www.R-project.org/.

Riederer P, Weiser M, Wichart I, Schmidt B, Killian W, Rett A (1986). Preliminary brain autopsy findings in progredient Rett síndrome. Am J Med Genet Suppl 1: 305-315.

Rodriguez A, Ehlenberger DB, Dickstein DL, Hof PR, Wearne SL (2008). Automated three-dimensional detection and shape classification of dendritic spines from fluorescence microscopy images. PLoS ONE 3: e1997.

Rodriguez A, Ehlenberger DB, Hof PR, Wearne SL (2006). Rayburst sampling, an algorithm for automated three-dimensional shape analysis from laser scanning microscopy images. Nat Protoc 1: 2152-2161.

Roux JC, Dura E, Moncla A, Mancini J, Villard L (2007). Treatment with desipramine improves breathing and survival in a mouse model for Rett syndrome. Eur J Neurosci 25: 1915-1922.

Samaco RC, Mandel-Brehm C, Chao HT, Ward CS, Fyffe-Maricich SL, Ren J et al (2009). Loss of MeCP2 in aminergic neurons causes cellautonomous defects in neurotransmitter synthesis and specific behavioral abnormalities. Proc Natl Acad Sci USA 106: 21966-21971.

Santini E, Alcacer C, Cacciatore S, Heiman M, Hervé D, Greengard P et al (2009). L-DOPA activates ERK signaling and phosphorylates histone $\mathrm{H} 3$ in the striatonigral medium spiny neurons of hemiparkinsonian mice. J Neurochem 108: 621-633.

Sholl DA (1953). Dendritic organization in the neurons of the visual and motor cortices of the cat. J Anat 87: 387-406.

Tandé D, Höglinger G, Debeir T, Freundlieb N, Hirsch EC, François C (2006). New striatal dopamine neurons in MPTPtreated macaques result from a phenotypic shift and not neurogenesis. Brain 129: 1194-1200.

Viemari JC, Roux JC, Tryba AK, Saywell V, Burnet H, Pena F et al (2005). Mecp2 deficiency disrupts norepinephrine and respiratory systems in mice. J Neurosci 25: 11521-11530.

Wenk GL, Naidu S, Casanova MF, Kitt CA, Moser H (1991). Altered neurochemical markers in Rett's syndrome. Neurology 41: $1753-1756$

(c) (1) (8) This work is licensed under a Creative Commons Attribution-NonCommercial-NoDerivs 3.0 Unported License. To view a copy of this license, visit http:// creativecommons.org/licenses/by-nc-nd/3.0/

Supplementary Information accompanies the paper on the Neuropsychopharmacology website (http://www.nature.com/npp) 